\title{
Cortical thickness increases after simultaneous interpretation training
}

\author{
Alexis Hervais-Adelman1, Barbara Moser-Mercer ${ }^{2}$, Micah M. Murray ${ }^{3-6}$, and Narly \\ Golestani $^{{ }^{1, *}}$
}

1. Brain and Language Lab, Department of Clinical Neuroscience, University of Geneva, Switzerland

2. InZone, University of Geneva, Switzerland

3. The Laboratory for Investigative Neurophysiology (The LINE), Departments of Clinical Neurosciences and Radiology, University Hospital Centreand University of Lausanne, Lausanne, Switzerland

4. The EEG Brain Mapping Core, Centre for Biomedical Imaging (CIBM), Lausanne, Switzerland

5. The Department of Ophthalmology, Jules Gonin Eye Hospital, University of Lausanne, Switzerland

6. The Department of Hearing and Speech Sciences, Vanderbilt University, Nashville, TN, USA

\footnotetext{
* Corresponding Author

Brain and Language Lab

Campus Biotech

Neuroscience Department

9, Chemin des Mines
}

1202 Geneva

Tel: +41223795352

E-mail: Narly.Golestani@unige.ch 
Author Contributions: N.G. conceived and supervised the study. A.H.-A. carried out the analyses. A.H.-A and N.G. collected data and wrote the paper, and B.M.M and M.M. contributed to the paper. 


\begin{abstract}
Simultaneous interpretation is a complex cognitive task that not only demands multilingual language processing, but also requires application of extreme levels of domain-general cognitive control. We used MRI to longitudinally measure cortical thickness in simultaneous interpretation trainees before and after a Master's program in conference interpreting. We compared them to multilingual control participants scanned at the same interval of time.Increases in cortical thickness were specific to trainee interpreters. Increases were observed in regions involved in lower-level, phonetic processing(left posterior superior temporal gyrus, anterior supramarginal gyrus and planum temporale), in the higher-level formulation of propositional speech (right angular gyrus) and in the conversion of items from working memory into a sequence (right dorsal premotor cortex), and finally, in domain-general executive control and attention (right parietal lobule). Findings are consistent with the linguistic requirements of simultaneous interpretation and also with the more general cognitive demands on attentional control for expert performance in simultaneous interpreting.Our findings may also reflect beneficial, potentially protective effects of simultaneous interpretation training, which has previously been shown to confer enhanced skills in certain executive and attentional domains over and above those conferred by bilingualism.
\end{abstract}




\section{Keywords}

Cortical thickness; plasticity; simultaneous interpreting; training; left superior temporal gyrus; left supramarginal gyrus; structural MRl; multilingualism

\section{Introduction}

There is growing interest in understanding the brain's structural changes, or plasticity, arising from bilingualism (García-Pentón, Pérez Fernández, Iturria-Medina, Gillon-Dowens, \& Carreiras, 2014; Klein, Mok, Chen, \& Watkins, 2014; Li, Legault, \& Litcofsky, 2014; Mechelli et al., 2004; Ressel et al., 2012; Stein et al., 2012) and from training in language-specific domains,such as phonetics(Golestani, Price, \& Scott, 2011; Vandermosten, Price, \& Golestani, 2015) and simultaneous interpreting(Elmer, Hanggi, \& Jancke, 2014; Elmer, Hanggi, Meyer, \& Jancke, 2011). A growing number of cross-sectional (Klein, et al., 2014; Li, et al., 2014; Mechelli, et al., 2004; Olulade et al., 2015; Ressel, et al., 2012) as well as some longitudinal (Schlegel, Rudelson, \& Tse, 2012; Stein, et al., 2012) studies on bilingualism have reported structural findings in regions including the left inferior parietal cortex, the auditory cortex, the inferior frontal gyri bilaterally and in regions involved in polyglot language control (Abutalebi \& Green, 2007, 2008; Hervais-Adelman, Moser-Mercer, \& Golestani, 2011). However, the results are relatively heterogeneous and diverse (Golestani, 2014), perhaps due to differences in populations, brain imaging sequences and brain imaging analysis approaches across studies(García-Pentón, Fernández García, Costello, Duñabeitia, \& Carreiras, 2015; Higby, Kim, \& Obler, 2013).

Beyond work on language acquisition and bilingualism, previous work on language expertise has shown grey (Golestani, et al., 2011)and white matter (Vandermosten, et al., 2015)differences between phonetics experts and controls in the auditory cortices bilaterally and in the left pars opercularis. These regions belong to the dorsal, audio-motor stream that subserves audio-motor mapping of sounds onto articulatory-based representations, rather than to the ventral meaning integration interface(Golestani, 2015; Golestani \& Pallier, 2007; Hickok \& Poeppel, 2004, 2007; Rodriguez-Fornells, Cunillera, Mestres-Misse, \& de Diego-Balaguer, 2009; Saur et al., 2008). Moreover,among the phoneticians there was a correlation between years of phonetics transcription training and grey and white matter properties of these regions, suggesting experience-dependent plasticity in relation to this relatively low-level form of linguistic expertise(Golestani, et al., 2011; Vandermosten, et al., 2015).

Simultaneous interpreting (SI), by contrast, is a linguistic task that involves higher-level (i.e. phonetic but also semantic, syntactic and prosodic) linguistic processing and extensively taps cognitive control mechanisms that are not specifically linguistic in nature. Listening to continuous prose in one language while simultaneously converting and producing the narrative in another language requires real-time processing of a source input, whilst simultaneously transposing the content of that input to a target language, monitoring production of that output, whilst 
maintaining access to and control over the relevant phonetic, semantic, syntactic and prosodic rules of both source and target languages. Interpreters listen to source language input while producing target language outputfor an average of $65 \%$ of their time on task. In order to monitor their output in the target language interpreters need to listen to it, while simultaneously listening to newly arriving input in the source language. Thus, success at simultaneous interpretation depends not only on outstanding processing speed and excellent verbal working memory skills, but also on the ability to simultaneously comprehend and produce speech in two oftentimes structurally very dissimilar languages, while also monitoring one's output and continuously translating it in real-time (Moser-Mercer, Lambert, Daró, \& Williams, 1997). Consistent with this, a recent functional magnetic resonance imaging (fMRI) study has shown that simultaneous interpretation recruits brain networks associated with speech comprehension and production such as the left inferior frontal gyrus, auditory and posterior temporal and temporo-parietal regions, alongside regions involved in more domain-general functions such as task-switching, conflict resolution, and inhibition; functionsthat have previously been implicated in languagecontrol. These latter regions included the anterior cingulate cortex and a thalamo-striatal-cerebellar network (Hervais-Adelman, Moser-Mercer, Michel, \& Golestani, 2014).

Two recentstructural brainimaging studies haveinvestigated grey (Elmer, et al., 2014) and white matter (Elmer, et al., 2011) in professional simultaneous interpreters.Unlike the trainee interpreters studied by Hervais-Adelman and colleagues $(2014,2015)$ described above, these two studies featured highly experienced professionals. Elmer and colleagues (Elmer, et al., 2014)revealed lower grey matter volumes in the interpreters compared to control participants in the left pars opercularis andsupramarginal gyrus (SMG), in the middle-anterior cingulate gyrus, and bilaterally in the pars triangularis and middle-anterior insula. Within the interpreters groups, the grey matter volume in a subset of these regions (the left pars triangularis, right pars opercularis and middle-anterior cingulate gyrus) and in the bilateral caudate nucleus was negatively correlated with the cumulative number of interpreting hours. These findings likely reflect experience-dependent structural plasticity in these language-related and cognitivecontrol regions, although some of the differences may have predated the training (Elmer, et al., 2014) and may thus reflect possibly innate, domain-specific aptitudes (c.f. Golestani, et al., 2011).

In the present study, we examined cortical thickness changes arising from simultaneous interpretation training longitudinally, before and after our participants undertooka Master's programme in conference interpreting. The longitudinal nature of our design allows for greater sensitivity to training-related changes, and mitigates against many confounding effects of cohort(Fitzmaurice, Laird, \& Ware, 2012). Due to the fact that many studies have reported positive relationships between behavioural measures and regional measures of thickness and/or volume (Blackmon et al., 2010; Foster \& Zatorre, 2010; Golestani, 2014; Li, et al., 2014; Wong, Ettlinger, 
Sheppard, Gunasekera, \& Dhar, 2010), we predicted that we would find trainingrelated increases in cortical thickness in brain regions responsible for speech production and comprehension, in onesinvolved in language and cognitive control, and in attentional regions. These include fronto-temporo-parietal regions, motor and premotor regions, the anterior cingulate gyrus and subcortical regions, and right superior parietal attentional regions. We expected structural modifications to at least partly converge in terms of localisation with brain regions previously found to be functionally involved in simultaneous interpreting(Hervais-Adelman, et al., 2014) and in language control (Hervais-Adelman et al, 2011), and in regions found to differ structurally between interpreters and controls by others (Elmer, et al., 2014; Elmer, et al., 2011).

\section{Methods}

\subsection{Participants}

Sixty-seven individuals participated in the study. Of these, 34 were trainee interpreters, and 33 constituted a control group. For logistical reasons, participants were scanned at either of two imaging centres (see below), but all scans for a given individual always took place on the same scanner / at the same centre. The trainee interpreters were enrolled in the Master's program in conference interpreting at the Faculty of Translation and Interpreting at the University of Geneva, Switzerland. The control participants were university students undertaking post-graduate studies in a range of disciplines other than the fields of interpretation, translation or modern languages. All participants were required to be multilingual and reported mastering a minimum of three languages (Table 1). Research was carried out in accordance with the Declaration of Helsinki and approved by the research ethics committeesof the Lausanne and Geneva University Hospitals.Participants gave informed consent and were free to withdraw from the study at any time. They were remunerated for their participation.

Characteristic data associated with the two groups are shown in Table 1. The groups were matched for age andgender. Both groups contained a small number of left-handed individuals(assessed using the Edinburgh handedness inventory Oldfield, 1971).Since trainee interpreters are a scarce population, we elected to be inclusive in our participant selection, and to account for this by including a matched proportion of left-handed individuals in the control group.It should be noted that since this is primarily a within-subjects design, we would not expect any particular influence of handedness on the results, especially since the proportions are matched across groups.

At baseline, both groups were scored on a compound measure of language experience and proficiency, as assessed by interview. This measure is described in Golestani and colleagues (2011), and was calculated as follows: knowledge of each 
of the participant's languages was weighted according to the age at which it had been learnt (Age of Acquisition, or AoA, higher weight for languages learnt earlier in life) and according to the fluency with which it was spoken (higher weight for more fluently spoken languages). The following weights were used: 1) proficiency: not fluent $=1$, somewhat fluent $=2$, moderately fluent $=3$, quite fluent $=4$, very fluent $=5$,

native $=6 ; 2$ ) age of acquisition: ages $\geq 21=1$, ages $13-20=2$, ages $7-12=3$, ages $1-6$ $=4$, at birth $=5$. The sum of the weightedAoA and fluency factors across all of a participant's reported languages was taken as an aggregate measure of their language experience and proficiency (LEXP). The groups differed slightly on LEXP (Controls: 32.94 , Interpreters: 37.18 , unpaired $t_{(65)}=2.01, p=.048$ ). This effect was driven by a difference in the ages of acquisition of the reported languages, with the trainee interpreters, on average, having begun to acquire more languages earlier. The groups did not differ in terms of their cumulative self-rated proficiency (see Table 1), nor did they differ in terms of the numbers of languages they reported speaking.

Given reports in the literature on the impact of age of acquisition on brain structure in bilingual vs monolingual populations (e.g. Klein, et al., 2014), we checked whether the two groups differed in terms of age of acquisition of their first foreign language. The two groups were divided into subgroups of "simultaneous" (i.e. in the first year of life), "early" (up to 6 years old) and "late" (after 6 years old) bilinguals (disregarding the age of acquisition of their third and subsequent languages). The distribution of participants into these bins (see Table 1) was compared using a chi-squared test and found not to be significantly different $\left(\chi^{2}=1.86, p=.394\right)$.

\subsection{Structural MRI}

Participants were scanned on either of two separate scanners of the same model, using identical acquisition parameters. Scan-rescan reliability of MPRAGE sequences has been demonstrated to be high (Wonderlick et al., 2009). However,given that hardware differences may influence acquisition, we nonetheless controlled for scanner effects at the analysis stage.

\subsubsection{Site 1 data acquisition}

Twelve trainees and 13 control participants were scanned using a Siemens 3T Trio MRI scanner, fitted with an 8-channel head-coil, at the Centre for Biomedical Imaging (CIBM) within the Radiology Department of the Centre Hospitalier Universitaire Vaudois (CHUV) Lausanne, Switzerland. T1 images were acquired using an MPRAGE sequence with the following parameters: Sagittal orientation, FoV: $240 * 256$, slice thickness $1.2 \mathrm{~mm}, 1 \mathrm{~mm} * 1 \mathrm{~mm}$ in-plane resolution, TR $2400 \mathrm{~ms}$, TE $2.98 \mathrm{~ms}$, nPhase Encoding steps: 239, Flip angle $9^{\circ}$. 


\subsubsection{Site 2 data acquisition}

Twenty-two trainees and 20 control participants were also scanned on a Siemens 3T Trio MRI scanner,fitted with an 8-channel head-coil,at the Brain and Behaviour Lab (BBL), University of Geneva, Geneva, Switzerland. T1 images were acquired using an MPRAGE sequence with the same parameters as above. These participants also completed a longitudinal functional imaging investigation on the neural bases of simultaneous interpreting (Hervais-Adelman, Moser-Mercer, \& Golestani, 2015; Hervais-Adelman, et al., 2014), for which data were acquired during the same scanning session.

\subsection{Simultaneous interpretation training}

The trainee participants were enrolled in the Master's program in conference interpreting at the Faculty of Translation and Interpreting of the University of Geneva. The course consists of 15 months of intensive training, spread over three 14week semesters. A first semester in consecutive interpreting (interpretation commences once the speaker has finished, with the interpreter thus not listening and speaking simultaneously), is followed by two semesters during which consecutive interpreting is taught alongside simultaneous interpreting (see task description above). Teaching covers a variety of aspects of the profession, incorporating strands such as theory of interpreting and conference terminology, alongside ongoing development of the practical skills of interpreting gained through deliberatepractice. Trainees receive formal instruction in simultaneous interpreting in their respective language combinations for at least 10 hours a week. In addition, they are expected to practice independently. Instruction and independent practice take different forms, with the former consisting of master classes and closelysupervised sessions tailored to each student's language combination, as well as tutor-supervised group practice. Individual practice may be with a peer group, who provide feedback on performance, or it may be solitary, where trainees record their interpreting performance, which they subsequently assess. Trainees undergo continuous assessment during the course. They are regularly reminded of the relevant learning objectives, and of the weaknesses that they should aim to address through deliberate practice, a type of practice that focuses on specific skill deficits, usually one at a time. No quantitative suggestions are made as to the amount of individual practice to undertake, but rather the emphasis is placed on reaching progressively more challenging learning outcomes.

Although not all of the participants passed all their final exams at their first attempt, their continuous assessment revealed that all displayed a learning trajectory that matched or exceeded the expected level of progress for a trainee at that stage of the training program. Indeed, any trainee consistently failing to meet the expected standard did not remain enrolled in the course. Thus, at the end of their course they were deemed to have reached a level of capability that, with appropriate diligence and discipline, would allow them to pass their exams, and, 
eventually, permit them to enter the profession. Due to the nature of students' evaluation during the course, no straightforward measures of learning outcomes, beyond successful completion, are available. Since this research is concerned with examining the changes in the brain that may be related to the development of these skills, an inclusion criterion was that trainee interpreters must reach the end of their course and be admitted to sit their final exams.Control participants had no formal interpretation or translation experience, despite being multilingual.

\subsection{Image Processing}

Cortical reconstruction and volumetric segmentation was performed with the Freesurfer image analysis suite (version 5.3.0), which is documented and freely available for download online (http://surfer.nmr.mgh.harvard.edu/). The technical details of these procedures are described in prior publications (full details may be found in Reuter, Schmansky, Rosas, \& Fischl, 2012). We exploited Freesurfer's capability of extracting cortical surfaces to enable analysis of cortical thickness changes with training.

To extract reliable thickness estimates, images were automatically processed with the longitudinal stream(Reuter, et al., 2012). Specifically, unbiased withinsubject template spaces and imageswere created using robust, inverse consistent registration (Reuter, et al., 2012). Several processing steps, such as skull stripping, Talairach transforms, atlas registration as well as spherical surface maps and parcellations were then initialized with common information from the within-subject template, significantly increasing reliability and statistical power(Reuter, et al., 2012). Freesurfer executes fully automated segmentation, and provides measures not only of cortical thickness but also of mean curvature. The quality of the segmentations was estimated by calculating the proportion of surface vertices that had extremely outlying estimated curvature values (greater than 2.5 standard deviations from the group mean), which would be indicative of poor segmentation performance, resulting fromneuroanatomically implausible discontinuities or "spikes" in the estimated surface segmentation. A threshold of $1 \%$ was set as an exclusion criterion, which all processed images passed. The processed cortical surface images were then smoothed using a $5 \mathrm{~mm}$ surface-based kernel(Hagler, Saygin, \& Sereno, 2006), and analysed.

\subsection{Analysis}

Extracted cortical thickness values were analysed to determine whether there were any changes that arose from training. Freesurfer was used to calculate the annualised symmetrised percent change in cortical thickness ${ }^{1}$ at each vertex for each participant. This measure reflects the total change in thickness, scaled by the

\footnotetext{
${ }^{1}$ Symmetrised percent change is calculated as follows: (Thickness at Time2 - Thickness at Time1/interval in years)/(0.5* [Thickness at Time $2+$ Thickness at Time 1$])$.
} 
interscan interval, expressed as a percentage of mean cortical thickness across both timepoints. It thus standardises both for between-subject differences in cortical thickness, and for different inter-scan intervals across participants. Symmetrised percent change was then analysed to seek between-group differences that would reflect the effect of simultaneous interpreting training.

Analyses were carried out using FreeSurfer's mri_glmfit utility to estimate a general linear model incorporating a two-level between-subjects factor of group (Interpreter, Control), with categorical covariates of Sex, Scanner and Handedness, and a continuous covariate of Age.The parameter estimates for the two groups were contrasted to determine if any regions showed differing rates of thickness change as a function of group. We report results that meet a significance level of $p<.0001$, uncorrected for multiple comparisons. Where relevant, we indicate results that meet thresholds for significance corrected for multiple comparisons at the vertex level using FDR at $p<.05$ or at the cluster level FWE at $p<.05$ using a cluster-forming threshold of $p<.0005$ (see Fig. 1 and Table 2). Given the longitudinal nature of the design, which by definition controls for individual differences, and given that we did not expect (nor did we assess)significant changes in language proficiency across the two time points (the interpreters were fluent in the languages that they worked to and from beforeentering the Master's program, and none reported adding a new working language between the two assessment points), we did not include LEXP, nor its AoA constituent, as a covariate in this analysis. Since we are sensitive to potential concerns arising from this, we present the analysis conducted here with the inclusion of the AoA scores in Supplementary Figure 1 and Supplementary Table 1. The results are qualitativelyindistinguishable from those presented here.

\section{Results}

Results revealed group differences in the change in cortical thickness in three posteriorperi-Sylvian regions of the left-hemisphere,namely, the posterior superior temporal gyrus, the supramarginal gyrus and the planum temporale within the posterior lateral(or Sylvian) fissure. Group differences in the change in cortical thickness were also found in the right hemisphere, in the superior parietal lobule, in the angular gyrus of the inferior parietal cortex, in the intraparietal sulcus and in the superior frontal gyrus. The results are illustrated in Figure 1, and detailed in Table 2.

Inspection of the nature of the effects (see plots in Figure 1) reveals that in all cases, an increase in cortical thickness over time occurred in the trainee interpreters, while a decrease occurred in the control participants. As age-related decreases in cortical thickness may be expected in adult populations, we also checked for a main effect of time on cortical thickness. A number of regions showed significant decreases in cortical thickness over both groups, notably the middle cingulate cortex and the middle to anterior superior temporal sulcus in the left hemisphere. However, while these effects are of some interest, we choose to only discuss findings that can convincingly be ascribed to training. We therefore confine our 
discussion to results that show a significant between-group difference. For the sake of completeness, the within-group effects of time are presented in Supplementary Figure 2 and the main-effect of time in Supplementary Figure 3 and Supplementary Table 2.

The left-hemisphere regions (STG, SMG and lateral fissure) are located within the wider set of regions that have previously been found to be functionally implicated in simultaneous interpreting in a subset (42 participants) of this sample (Hervais-Adelman, et al., 2014, see Figure 1 panel B for an overview of these results). Among the regions found to show an effect of training in the right hemisphere, only the superior frontal gyrus has previously been functionally implicated in simultaneous interpreting.

The longitudinal design and the use of symmetrised percent change should minimise the impact of any pre-training group differences. We nonetheless tested for the presence of pre-training differences in cortical thickness, which could, for example, arise from group differencesin language proficiency. Consistent with the heterogeneity of the published literature on structural changes in multilingual individuals (García-Pentón, et al., 2015), no significant differences were revealed between the two groups despite the difference in LEXP.

\section{Discussion}

Our results revealed cortical thickness changes, arising from approximately 14 months of simultaneous interpretation training, in a number ofregions across both cerebral hemispheres. Increases in cortical thickness were observed in left perisylvian regions generally associated with speech processing,ones that have previously been found to be functionally implicated in SI. Training-related cortical thickness changes in the right hemispherewere not confined to regions functionally implicated in $\mathrm{SI}$, implicating an even broader network in the development of SI expertise.

In the interpretation trainees, we observed increases in cortical thicknessin the upper bank of the left posterior superior temporal sulcus extending into the superior temporal gyrus, in the ventral anterior left supramarginal gyrus (immediately medial to the left parietal operculum) and in the left planum temporale within the posterior lateral fissure. These regions are part of the dorsal audio-motor stream, involved in mapping speech sounds onto articulatory representations(Hickok \& Poeppel, 2007; Scott, McGettigan, \& Eisner, 2009). The left mid to posterior STS is posited to be at the interface of the ventral and dorsal streams as a hub for phonological processing, which, after initial spectrotemporal analysis in the planum temporale, is involved in mapping speech sounds both onto articulatory representations (within the dorsal stream) and onto lexical ones (within the ventral stream) (Griffiths \& Warren, 2002; Hickok \& Poeppel, 2007). The planum temporale and parietal operculumare thought to integrate predictive feedforward and post-articulatory sensory feedback signals during articulation (Guenther, 2006), 
and it has been shown that there is greater activation in these regions during speech production in the second compared to the first language of bilinguals(Simmonds, Wise, Dhanjal, \& Leech, 2011). Indeed, for successful performance, interpreters are required to simultaneously handle the phonotactics of both an incoming stream and of an outgoing one, which necessitates simultaneous or quasi-simultaneous access to and control of two phonemic inventories, and thus potentially induces cortical thickening in these regions. In addition, it is plausible that the structural changes observed in these lower-level, sensori-motor and phonological regions arise from the requirement of attending to speech input in the source language while also attending to one's own speech output in the target language during simultaneous interpretation. That is, they may be due to the phonological requirements of processing two simultaneouslyincoming streams of speech, one being the utterances to be interpreted and the other being one's own voice.

In the right hemisphere, we observed increased cortical thickness arising from interpretation training in the superior parietal lobe, the intraparietal sulcus and the adjacent angular gyrus, as well as in the posterior superior frontal gyrus. The right superior parietal lobule (BA7P) isconsidered part of the dorsal attentional system (Corbetta \& Shulman, 2002). While this region was not specifically functionally engaged during the interpreting task executed in Hervais-Adelman et al.'s previous studies by novices(i.e. before any SI training Hervais-Adelman, MoserMercer, Michel, \& Golestani, 2015), it is possible that the attentional control processes required by interpreting are more intensively, or differently, invoked at a later stage of learning, once the materials being interpreted become more challenging, or during deliberate, reflective practice. The right intraparietal sulcus has been associated with executive control (Collette, Hogge, Salmon, \& Van der Linden, 2006; Seeley et al., 2007) as well as with non-spatial working memory and attention (Coull \& Frith, 1998); these functions are also recruitedfor simultaneous interpreting.

The right angular gyrus has been shown to be involved in the production of propositional speech, i.e. speech for which the speaker is required to formulate a message, in contrast to simple repetition or reproduction of over-learned sequences such as nursery rhymes(Blank, Scott, Murphy, Warburton, \& Wise, 2002). This is particularly relevant to interpreting, because in order to produce high quality interpretations the interpreter will need to have not only translated but also reformulated the content of the incoming speech. The fact that functional recruitment of this region was not shown in the previous studies may be due to the fact that the materials used by Hervais-Adelman et al.(2014, 2015)for the participants to interpret during scanning were too simple to elicit such a requirement, or that naïve interpreters do not extensively reformulate content.

The region of the right superior frontal gyrus showing cortical thickening in the trained interpreters was previously shown to be engaged by $\mathrm{SI}$, although as part of a larger swath of activation spreading to brain regions responsible for 
sensorimotor processing (Hervais-Adelman et al, 2014). More specifically, this region of SFG sits within the dorsal premotor cortex (BA6). This region has been ascribed a role in various cognitive tasks (Abe \& Hanakawa, 2009), although of notable importance in the context of $\mathrm{SI}$ is the evidence that this region is implicated in converting items in working memory into a sequence (Hanakawa et al., 2002).

The fact that structural changes appear to have occurred in several brain regions (the right superior parietal lobule, the intraparietal sulcus and angular gyrus) outside of those previously shown to be functionally recruited by SI (HervaisAdelman, et al., 2014) can be due to a number of reasons. First, there likely exist non-linear relationships between brain function and structure. Second, functional and structural changes are likely to have different time courses, and the relationship between these may depend on the region at hand. Third, the previous functional imaging study assessed task performance using stimuli that were simpler and shorter than the material that the interpreters were trained on over the Master's course.

Regions in which differences in symmetrised percent change were found between the groups showed a pattern of thickening in the trainee interpreters and thinning in the controls. Cortical thickening is often associated with training in many domains (e.g. Draganski \& May, 2008; Engvig et al., 2010; Wei, Zhang, Jiang, \& Luo, 2011; Wenger et al., 2012), while cortical thinning is an age-related change that is expected to occur over time (Salat et al., 2004).The mean rate of cortical thinning that we observed is numerically greater than in some previous reports on lifespan decreases in cortical thickness. For example, in a recent paper on cortical changes across the adult lifespan (Storsve et al., 2014), annual decreases in cortical thickness of up to $0.7 \%$ are reported (varying across different brain regions), where in our controls the annualized symmetrized percent change ranges from approximately 1 $2.5 \%$. The reason for the discrepancy may be related to the age-range examined in our study, and/or to other factors such as possibly greater rates of training-related change (increases in some regions and concurrent decreases in other regions) in our participants who, unlike the majority of the general population, were actively engaged in learning or training. If active engagement in training or the acquisition of expertise leads to a greater degree of cortical plasticity over a given time period, this may, in part, account for these findings. However, it is important to note that in the regions where there is a group-wise difference, the rate of thinning is not significantly greater than zero, at a threshold of $p<.0001$ (see Supplementary Figure 2 for a complete illustration of the cortical changes within each group).

Supplementary Figure $2 \mathrm{C}$ also illustrates that the region of pSTS, where a significant impact of training is observed on SPC, also shows a reliable within-group effect of thickening at uncorrected $p<.0001$.. Consistent with previous work on learning and experience-dependent plasticity(Engvig, et al., 2010; Golestani, et al., 2011; Martensson et al., 2012; Metzler-Baddeley, Caeyenberghs, Foley, \& Jones, 2016), this is likely to arise from the relatively long-term training undergone by the interpreters, which may be considered as conferring a "neuroprotective" effect 
insofar as it may prevent cortical thinning in these regions. Indeed, behavioural research has shown that simultaneous interpretation training may confer enhanced skills in some executive functions, over and above those conferred by bilingualism (Dong \& Liu, 2016; Morales, Padilla, Gomez-Ariza, \& Bajo, 2015; Padilla, Bajo, \& Macizo; Timarová et al., 2014; Yudes, Macizo, \& Bajo, 2011, 2012; Yudes, Macizo, Morales, \& Bajo, 2013).

Our structural plasticity findings converge partly with the cross-sectional ones obtained by Elmer and colleagues (Elmer, et al., 2014), in particular with regard to left SMG. Elmer and colleagues found structural differences in this same region, and also in the left pars opercularis, the left middle anterior cingulate gyrus, and in the bilateral pars triangularis and middle anterior insula(Elmer, et al., 2014). In the study by Elmer and colleagues, lower grey matter volumes were found in professional interpreters compared to multilingual controls in these regions, whereas we found increases in cortical thickness in left SMG following training. A related study used diffusion tensor imaging (DTI) to examine white matter connectivity in the same groups of interpreter and control participants (Elmer, et al., 2011). This study revealed decreased fractional anisotropy (FA) in the interpreter compared to control participants in fibre tracts underlying the left anterior insula, the upper corticospinal tract, the right inferior parietal lobe, and the right dorsal caudate nucleus. There was also lower FA in the interpreters in the genu, body and splenium of the corpus callosum. The authors interpreted these findings as reflecting training-related plasticity in interhemispheric connectivity, and white-matter connectivity between brain regions known to be important for sensory-motor mapping and speech articulation, functions known to be important for efficient interpretation (Elmer, et al., 2011). FA is often said to reflect microstructural integrity, with lower FA values reflecting poorer integrity. However, FA values derived from diffusion-weighted images are the result of a composite of microscopic and macroscopic factors, such as (but not limited to) intravoxel orientation of fibres, myelination, packing density, local complexity of fibre bundles and number of axons. While the notion of "integrity" may be a convenient shorthand, it is a misleading term (see Jones, Knosche, \& Turner, 2013 for a complete discussion of this issue and the many caveats associated with interpreting FA differences). Thus, changes in this measure can be said to reflect structural alterations, but the precise nature of these cannot be determined.

Several causes could account for the discrepancies in the location and direction of our findings and those of Elmer and colleagues. First, different measures were examined in the two studies (thickness here and volume in the previous study), and the direction of the relationship between brain anatomy and language skill can differ depending on which specific morphometric measure is being examined (García-Pentón, et al., 2015; Roehrich-Gascon, Small, \& Tremblay, 2015). Second, the participants in the present study had far less experience than those tested by Elmer and colleagues; those presented here had just finished their simultaneous 
interpretation training, whereas those tested by Elmer et al. were established professionals who had practiced their skills for years before participation in the brain imaging studies (Elmer, et al., 2014; Elmer, et al., 2011). Third, our study compared brain structure longitudinally in the same participants, whereas Elmer and colleagues compared brain structure in professional interpreters to control participants cross-sectionally. As such, and as they also note, some of their findings may be due to pre-existing differences between the groups. Furthermore, there was a systematic age difference between their control and professional interpreter groups, which may have had ramifications on the volumetric results. Finally, our participants were engaged not only in simultaneous but also in consecutive interpretation training during the Master's course, whereas presumably the experts in the studies by Elmer and colleagues were primarily engaged in simultaneous interpreting in their professional work. Although these two types of interpretinghave overlapping features, especially with regards to the multilingual language comprehension and production demands, the absence of simultaneity in consecutive interpreting renders it a categorically different task since it does not require the same degree of online language control as does SI. It does however, have other demands, and we cannot rule out that these could have contributed to some changes we observe here.More generally, although many studies that examined brain structure in relation to language-related training have shown increases in grey or white matter volume(Golestani, et al., 2011; Klein, et al., 2014; Li, et al., 2014; Mechelli, et al., 2004; Ressel, et al., 2012; Stein, et al., 2012) or connectivity(GarcíaPentón, et al., 2014; Vandermosten, et al., 2015) related to training, others have shown differences in the opposite direction (Golestani, 2014; Marie \& Golestani, In Press; Olulade, et al., 2015; Porter, Collins, Muetzel, Lim, \& Luciana, 2011; RoehrichGascon, et al., 2015), again, possibly reflecting experience-dependent pruning or neural reorganisation. Nonetheless, the results reported here point to traininginduced cortical thickening. This may reflect that training- or experience-dependent changes may follow an "inverted- $U$ " shaped pattern over time, whereby initial increases in volume are followed by pruning (for review, see Lovden, Wenger, Martensson, Lindenberger, \& Backman, 2013).

In summary, our longitudinal findings point to cortical thickening following simultaneous interpretation training in brain regions known to be involved in audiomotor integration and phonological processing, in the formulation of propositional speech, in the conversion of items from working memory into a sequence and in executive control and attention. Our findings are consistent with the very broad linguistic and non-linguistic requirements of simultaneous interpreting, where not only lower-level, phonological processes but also higher-levelprocesses involved in speech formulation and working memory are solicited, along with domain-general, executive and attentionalones. More generally, it appears that training theseprocesses within the context of the online multilingual demands of SI may confer some protection against normal, age-related cortical thinning, at least in a 
subset of linguistic and attentional brain regions. Our findings are consistent with those of numerous studies on training-related plasticity, where structural changes have been shown to occur in brain regions that are functionally implicated in the domain of training at hand(Draganski et al., 2004; Imfeld, Oechslin, Meyer, Loenneker, \& Jancke, 2009; James et al., 2013; Maguire et al., 2000; Stein, et al., 2012; Tu et al., 2015). These changes likely depend on a number of factors, including but not limited to:the brain structural measures being examined, the age of participants, domain-specific aptitudes, and motivational and attentional factors. The present results suggest that the development of expertise in simultaneous interpreting tunes a speech-related brain network implicated in speech-sound processing, and a set of brain regions implicated in the executive control of attentional resources, alongside regions related to working memory. The changes we report here provide some circumstantial evidence for the potential cerebral basis of the cognitive advantages reported in professional interpreters relative to multilingual controls. Further longitudinal work over longer periods oftraining and of professional work in simultaneous interpretation, along with functional brain imaging and with measures of performance on linguistic and non-linguistic tasks can help to further understand the improvements underlying this form of linguistic expertise, and the time course, localisation and magnitude of the brain structural plasticity that accompanies this training. 


\section{Figures}

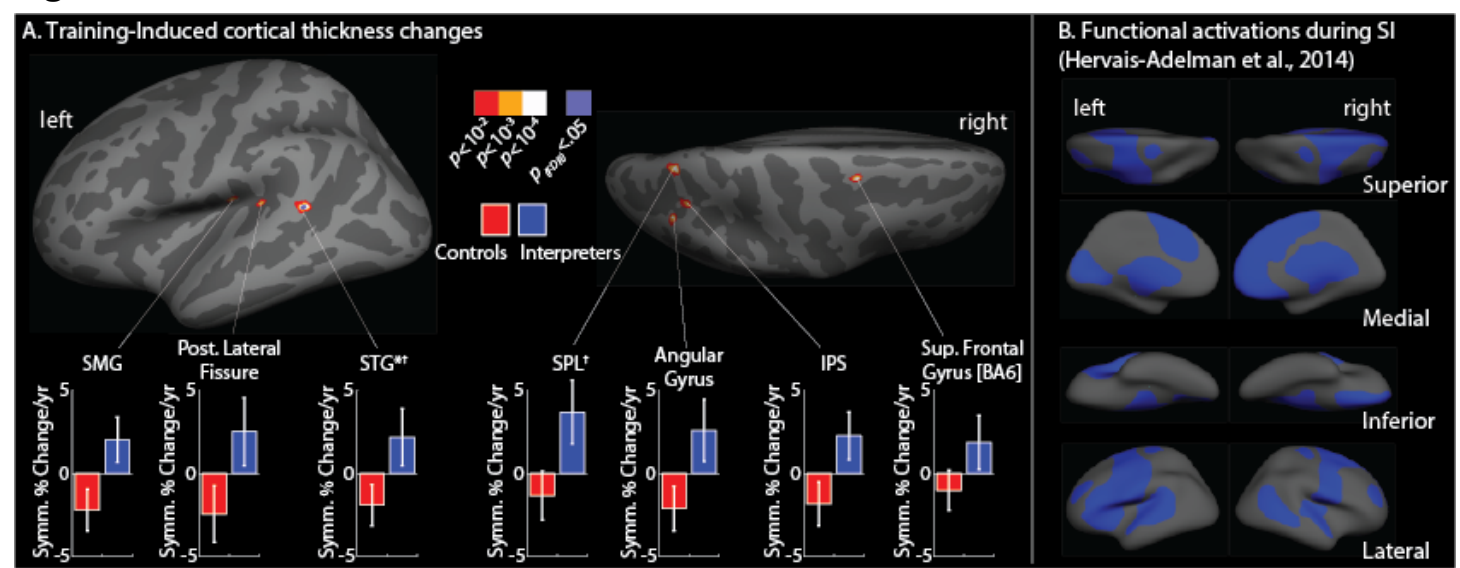

Figure 1: Panel A. Regions showing significant main effect of group in mean percent annualised cortical thickness change, projected on a canonical inflated white-matter surface. Dark patches represent sulci, light patches represent gyri. Note that for clarity of display, the clusters having reached significance (at $p<.0001$ ) are displayed at a threshold of $p<.01$. Colour coding indicates significance level. *Denotes clusters in which the peak reaches whole-brain FDR-corrected significance at $p<.05$, tdenotes clusters that reach whole-brain cluster-corrected significance at $p<.05$, with a cluster-forming threshold of $p<.0005$. Bar plots show symmetrised percent change for both groups at the peak co-ordinates of the cluster, error bars represent $95 \%$ Confidence Intervals. Panel B.For comparison, illustration of brain regions functionally recruited during simultaneous interpreting (data taken from HervaisAdelman, et al., 2014) projected onto the same inflated white-matter surface. 
Table 1:Characteristic information and comparison of control group and trainee interpreters. P-values reported for the between-groups comparisons are derived from chi-square or t-tests, as appropriate for ratios or continuous measures. * denotes a significant difference between the groups. Abbreviations: $\mathrm{N}=$ number, $\mathrm{F}=$ female, $\mathrm{M}=$ male, $\mathrm{LH}=$ left-handed, $\mathrm{RH}=$ right-handed, $\mathrm{NLang}=$ number of languages reported, LEXP = language experience and proficiency score, AoA =age of acquisition, Prof $=$ proficiency, $L 2 \mathrm{Acq}=$ age of acquisition of the first second language, $\mathrm{S}=$ simultaneous (in the first year after birth), $\mathrm{E}=$ early (up to six years old), $L=$ Late (after six years old)

\begin{tabular}{|l|l|r|r|r|l|r|r|r|r|r|l|}
\hline & N & F:M & LH:RH & $\begin{array}{l}\text { Site1: } \\
\text { Site2 }\end{array}$ & $\begin{array}{l}\text { Interscan } \\
\text { Interval in } \\
\text { years } \\
\text { (stdev) }\end{array}$ & $\begin{array}{l}\text { Age at } \\
\text { baseline } \\
\text { in years } \\
\text { (stdev) }\end{array}$ & $\begin{array}{l}\text { NLang } \\
\text { (stdev) }\end{array}$ & $\begin{array}{l}\text { LEXP } \\
\text { (stdev) }\end{array}$ & $\begin{array}{l}\text { Prof } \\
\text { Score } \\
\text { (stdev) }\end{array}$ & $\begin{array}{l}\text { AoA } \\
\text { Score } \\
\text { (stdev) }\end{array}$ & $\begin{array}{l}\text { L2 Acq } \\
\text { S:E:L }\end{array}$ \\
\hline Controls & 33 & $19: 14$ & $04: 29$ & $13: 20$ & $1.11(0.11)$ & $\begin{array}{r}25.7 \\
(5.27)\end{array}$ & $\begin{array}{r}4.09 \\
(1.35)\end{array}$ & $\begin{array}{r}32.94 \\
(8.86)\end{array}$ & $\begin{array}{r}13.63 \\
(3.48)\end{array}$ & $\begin{array}{r}19.30 \\
(5.82)\end{array}$ & $7: 10: 16$ \\
\hline SI Trainees & 34 & $19: 15$ & $03: 30$ & $12: 22$ & $1.13(0.06)$ & 26.03 & 4.62 & 37.18 & 14.88 & 22.35 & $12: 7: 15$ \\
$(4.39)$ & $(1.13)$ & $(8.37)$ & $(3.52)$ & $(5.15)$ & \\
\hline $\begin{array}{l}\text { Between- } \\
\text { groups } \\
\text { difference } \\
\text { (p) }\end{array}$ & - & .796 & .545 & .607 & .252 & .78 & .088 & $.048^{*}$ & .15 & $.027^{*}$ & .349 \\
\hline
\end{tabular}


Table 2: Clusters showing main effect of group in mean percent annualised cortical thickness change, with $5 \mathrm{~mm}$ smoothing. Clusters reported here contain peaks that reach a threshold for significance of $p<0.0001$.Abbreviations: Hem $=$ hemisphere, $\mathrm{R}=$ right, $L=$ left.*Denotes clusters in which the peak reaches whole-brain FDRcorrected significance at $p<.05$, tdenotes clusters that reach whole-brain clustercorrected significance at $p<.05$, with a cluster-forming threshold of $p<.0005$

\begin{tabular}{|c|c|c|c|c|}
\hline Hem & Region & $p$-value & $\begin{array}{l}\text { Size }\left(\mathrm{mm}^{\wedge} 2\right) \\
p<.0001\end{array}$ & $\begin{array}{l}x, y, z(m m \\
\text { MNI } 152 \\
\text { space) }\end{array}$ \\
\hline $\mathrm{L}$ & $\begin{array}{l}\text { Posterior Superior } \\
\text { Temporal Gyrus }\end{array}$ & 4.94E-08 & 40.72 & $-58,-45,11^{+}$ \\
\hline $\mathrm{R}$ & $\begin{array}{l}\text { Superior Parietal } \\
\text { Lobule [BA7P] }\end{array}$ & 4.57E-06 & 40.43 & $15,-70,53^{+}$ \\
\hline $\mathrm{R}$ & $\begin{array}{l}\text { Superior Frontal Gyrus } \\
\text { [BA6] }\end{array}$ & $1.33 \mathrm{E}-05$ & 11.15 & $23,1,60$ \\
\hline$L$ & Supramarginal Gyrus & $1.42 \mathrm{E}-05$ & 10.69 & $-41,-22,19$ \\
\hline$R$ & Angular Gyrus & $2.15 \mathrm{E}-05$ & 17.93 & $34,-72,39$ \\
\hline $\mathrm{R}$ & Intraparietal Sulcus & $2.71 \mathrm{E}-05$ & 8.96 & $29,-60,46$ \\
\hline $\mathrm{L}$ & Planum Temporale & $5.47 \mathrm{E}-05$ & 7.71 & $-39,-37,10$ \\
\hline
\end{tabular}


We wish to thank Professor Stephanie Clarke and Professor Reto Meuli for assistance in facilitating access to the scanner at the Centre for Biomedical Imaging (CIBM) at the Centre Hospitalier Universitaire Vaudois (cohort 1). We also wish to thank the Brain and Behaviour Lab at the University of Geneva for access to the scanner for cohort 2, as well as two anonymous reviewers for their helpful comments and suggestions for the paper. This work was supported by Swiss National Science Foundation grants awarded to N.G. (320030_122085 and PP00P3_133701) and to M.M.M (320030_149982 and 320030-169206).

\section{References}

Abe, M., \& Hanakawa, T. (2009). Functional coupling underlying motor and cognitive functions of the dorsal premotor cortex. Behav Brain Res, 198(1), 13-23. doi: 10.1016/j.bbr.2008.10.046

Abutalebi, J., \& Green, D. W. (2007). Bilingual language production: The neurocognition of language representation and control. Journal of Neurolinguistics, 20(3), 242-275.

Abutalebi, J., \& Green, D. W. (2008). Control mechanisms in bilingual language production: Neural evidence from language switching studies. Language and Cognitive Processes, 23(4), 557-582. doi: Doi 10.1080/01690960801920602

Blackmon, K., Barr, W. B., Kuzniecky, R., Dubois, J., Carlson, C., Quinn, B. T., . . . Thesen, T. (2010). Phonetically irregular word pronunciation and cortical thickness in the adult brain. Neuroimage, 51(4), 1453-1458. doi: 10.1016/j.neuroimage.2010.03.028

Blank, S. C., Scott, S. K., Murphy, K., Warburton, E., \& Wise, R. J. (2002). Speech production: Wernicke, Broca and beyond. [Research Support, Non-U.S. Gov't]. Brain, 125(Pt 8), 1829-1838.

Collette, F., Hogge, M., Salmon, E., \& Van der Linden, M. (2006). Exploration of the neural substrates of executive functioning by functional neuroimaging. Neuroscience, 139(1), 209-221.

Corbetta, M., \& Shulman, G. L. (2002). Control of goal-directed and stimulus-driven attention in the brain. Nat Rev Neurosci, 3(3), 201-215. doi: 10.1038/nrn755

Coull, J. T., \& Frith, C. D. (1998). Differential activation of right superior parietal cortex and intraparietal sulcus by spatial and nonspatial attention. Neuroimage, 8(2), 176-187. doi: 10.1006/nimg.1998.0354

Dong, Y., \& Liu, Y. (2016). Classes in Translating and Interpreting Produce Differential Gains in Switching and Updating. Front Psychol, 7, 1297. doi: 10.3389/fpsyg.2016.01297

Draganski, B., Gaser, C., Busch, V., Schuierer, G., Bogdahn, U., \& May, A. (2004). Neuroplasticity: changes in grey matter induced by training. Nature, 427(6972), 311-312. doi: 10.1038/427311a 
Draganski, B., \& May, A. (2008). Training-induced structural changes in the adult human brain. Behav Brain Res, 192(1), 137-142. doi:

10.1016/j.bbr.2008.02.015

Elmer, S., Hanggi, J., \& Jancke, L. (2014). Processing demands upon cognitive, linguistic, and articulatory functions promote grey matter plasticity in the adult multilingual brain: Insights from simultaneous interpreters. Cortex, 54, 179-189. doi: 10.1016/j.cortex.2014.02.014

Elmer, S., Hanggi, J., Meyer, M., \& Jancke, L. (2011). Differential language expertise related to white matter architecture in regions subserving sensory-motor coupling, articulation, and interhemispheric transfer. Hum Brain Mapp, 32(12), 2064-2074. doi: 10.1002/hbm.21169

Engvig, A., Fjell, A. M., Westlye, L. T., Moberget, T., Sundseth, O., Larsen, V. A., \& Walhovd, K. B. (2010). Effects of memory training on cortical thickness in the elderly. Neuroimage, 52(4), 1667-1676. doi:

10.1016/j.neuroimage.2010.05.041

Fitzmaurice, G. M., Laird, N. M., \& Ware, J. H. (2012). Applied longitudinal analysis (Vol. 998): John Wiley \& Sons.

Foster, N. E., \& Zatorre, R. J. (2010). Cortical structure predicts success in performing musical transformation judgments. Neuroimage, 53(1), 26-36. doi: 10.1016/j.neuroimage.2010.06.042

García-Pentón, L., Fernández García, Y., Costello, B., Duñabeitia, J. A., \& Carreiras, $M$. (2015). The neuroanatomy of bilingualism: how to turn a hazy view into the full picture. Language, Cognition and Neuroscience, 1-25. doi: 10.1080/23273798.2015.1068944

García-Pentón, L., Pérez Fernández, A., Iturria-Medina, Y., Gillon-Dowens, M., \& Carreiras, M. (2014). Anatomical connectivity changes in the bilingual brain. Neurolmage, 84, 495-504. doi: 10.1016/j.neuroimage.2013.08.064

Golestani, N. (2014). Brain structural correlates of individual differences at low to high levels of the language processing hierarchy: A review of new approaches to bilingualism research. The International Journal of Bilingualism, 18(1), 6-34. doi: doi:10.1177/1367006912456585

Golestani, N. (2015). Neuroimaging of phonetic perception in bilinguals. Bilingualism: Language and Cognition. doi: 10.1017/S1366728915000644

Golestani, N., \& Pallier, C. (2007). Anatomical correlates of foreign speech sound production. Cereb Cortex, 17(4), 929-934.

Golestani, N., Price, C. J., \& Scott, S. K. (2011). Born with an ear for dialects? Structural plasticity in the expert phonetician brain. J Neurosci, 31(11), 4213-4220. doi: 10.1523/JNEUROSCI.3891-10.2011

Griffiths, T. D., \& Warren, J. D. (2002). The planum temporale as a computational hub. Trends Neurosci, 25(7), 348-353.

Guenther, F. H. (2006). Cortical interactions underlying the production of speech sounds. Journal of Communication Disorders, 39(5), 350-365.

Hagler, D. J., Jr., Saygin, A. P., \& Sereno, M. I. (2006). Smoothing and cluster thresholding for cortical surface-based group analysis of fMRI data. Neuroimage, 33(4), 1093-1103. doi: 10.1016/j.neuroimage.2006.07.036

Hanakawa, T., Honda, M., Sawamoto, N., Okada, T., Yonekura, Y., Fukuyama, H., \& Shibasaki, H. (2002). The role of rostral Brodmann area 6 in mentaloperation tasks: an integrative neuroimaging approach. Cereb Cortex, 12(11), 1157-1170. 
Hervais-Adelman, A., Moser-Mercer, B., \& Golestani, N. (2011). Executive control of language in the bilingual brain: integrating the evidence from neuroimaging to neuropsychology. Front Psychol, 2, 234. doi: 10.3389/fpsyg.2011.00234

Hervais-Adelman, A., Moser-Mercer, B., \& Golestani, N. (2015). Brain functional plasticity associated with the emergence of expertise in extreme language control. Neuroimage, 114, 264-274. doi: 10.1016/j.neuroimage.2015.03.072

Hervais-Adelman, A., Moser-Mercer, B., Michel, C. M., \& Golestani, N. (2014). fMRI of Simultaneous Interpretation Reveals the Neural Basis of Extreme Language Control. Cereb Cortex. doi: 10.1093/cercor/bhu158

Hervais-Adelman, A., Moser-Mercer, B., Michel, C. M., \& Golestani, N. (2015). fMRI of Simultaneous Interpretation Reveals the Neural Basis of Extreme Language Control. Cereb Cortex, 25(12), 4727-4739. doi: 10.1093/cercor/bhu158

Hickok, G., \& Poeppel, D. (2004). Dorsal and ventral streams: a framework for understanding aspects of the functional anatomy of language. Cognition, 92(1-2), 67-99.

Hickok, G., \& Poeppel, D. (2007). The cortical organization of speech processing. Nat Rev Neurosci, 8(5), 393-402.

Higby, E., Kim, J., \& Obler, L. K. (2013). Multilingualism and the Brain. Annual Review of Applied Linguistics, 33, 68-101. doi: 10.1017/S0267190513000081

Imfeld, A., Oechslin, M. S., Meyer, M., Loenneker, T., \& Jancke, L. (2009). White matter plasticity in the corticospinal tract of musicians: a diffusion tensor imaging study. Neuroimage, 46(3), 600-607. doi: 10.1016/j.neuroimage.2009.02.025

James, C. E., Oechslin, M. S., Van De Ville, D., Hauert, C. A., Descloux, C., \& Lazeyras, F. (2013). Musical training intensity yields opposite effects on grey matter density in cognitive versus sensorimotor networks. Brain Struct Funct, 219(1), 353-366. doi: 10.1007/s00429-013-0504-z

Jones, D. K., Knosche, T. R., \& Turner, R. (2013). White matter integrity, fiber count, and other fallacies: the do's and don'ts of diffusion MRI. Neuroimage, 73, 239-254. doi: 10.1016/j.neuroimage.2012.06.081

Klein, D., Mok, K., Chen, J. K., \& Watkins, K. E. (2014). Age of language learning shapes brain structure: a cortical thickness study of bilingual and monolingual individuals. Brain Lang, 131, 20-24. doi: 10.1016/j.bandl.2013.05.014

Li, P., Legault, J., \& Litcofsky, K. A. (2014). Neuroplasticity as a function of second language learning: Anatomical changes in the human brain. Cortex, 58, 301324. doi: 10.1016/j.cortex.2014.05.001

Lovden, M., Wenger, E., Martensson, J., Lindenberger, U., \& Backman, L. (2013). Structural brain plasticity in adult learning and development. Neurosci Biobehav Rev, 37(9 Pt B), 2296-2310. doi: 10.1016/j.neubiorev.2013.02.014

Maguire, E. A., Gadian, D. G., Johnsrude, I. S., Good, C. D., Ashburner, J., Frackowiak, R. S., \& Frith, C. D. (2000). Navigation-related structural change in the hippocampi of taxi drivers. Proc Natl Acad Sci U S A, 97(8), 4398-4403. doi: 10.1073/pnas.070039597

Marie, D., \& Golestani, N. (In Press). Brain structural imaging of receptive speech and beyond: a review of current methods. 
Martensson, J., Eriksson, J., Bodammer, N. C., Lindgren, M., Johansson, M., Nyberg, L., \& Lovden, M. (2012). Growth of language-related brain areas after foreign language learning. Neuroimage, 63(1), 240-244. doi: 10.1016/j.neuroimage.2012.06.043

Mechelli, A., Crinion, J. T., Noppeney, U., O'Doherty, J., Ashburner, J., Frackowiak, R. S., \& Price, C. J. (2004). Neurolinguistics: structural plasticity in the bilingual brain. Nature, 431(7010), 757. doi: 10.1038/431757a

Metzler-Baddeley, C., Caeyenberghs, K., Foley, S., \& Jones, D. K. (2016). Task complexity and location specific changes of cortical thickness in executive and salience networks after working memory training. Neuroimage, 130, 48-62. doi: 10.1016/j.neuroimage.2016.01.007

Morales, J., Padilla, F., Gomez-Ariza, C. J., \& Bajo, M. T. (2015). Simultaneous interpretation selectively influences working memory and attentional networks. Acta Psychol (Amst), 155, 82-91. doi:

10.1016/j.actpsy.2014.12.004

Moser-Mercer, B., Lambert, S., Daró, V., \& Williams, S. (1997). Skill components in simultaneous interpreting. Paper presented at the Conference Interpreting: Current trends in research, Turku.

Oldfield, R. C. (1971). The assessment and analysis of handedness: the Edinburgh inventory. Neuropsychologia, 9(1), 97-113.

Olulade, O. A., Jamal, N. I., Koo, D. S., Perfetti, C. A., LaSasso, C., \& Eden, G. F. (2015). Neuroanatomical Evidence in Support of the Bilingual Advantage Theory. Cereb Cortex. doi: 10.1093/cercor/bhv152

Padilla, F., Bajo, M. T., \& Macizo, P. (2005). Articulatory suppression in language interpretation: Working memory capacity, dual tasking and word knowledge. Bilingualism-Language and Cognition, 8(3), 207-219. doi: 10.1017/S1366728905002269

Porter, J. N., Collins, P. F., Muetzel, R. L., Lim, K. O., \& Luciana, M. (2011). Associations between cortical thickness and verbal fluency in childhood, adolescence, and young adulthood. Neuroimage, 55(4), 1865-1877. doi: 10.1016/j.neuroimage.2011.01.018

Ressel, V., Pallier, C., Ventura-Campos, N., Diaz, B., Roessler, A., Avila, C., \& Sebastian-Galles, N. (2012). An effect of bilingualism on the auditory cortex. J Neurosci, 32(47), 16597-16601. doi: 10.1523/JNEUROSCI.1996-12.2012

Reuter, M., Schmansky, N. J., Rosas, H. D., \& Fischl, B. (2012). Within-subject template estimation for unbiased longitudinal image analysis. Neuroimage, 61(4), 1402-1418. doi: 10.1016/j.neuroimage.2012.02.084

Rodriguez-Fornells, A., Cunillera, T., Mestres-Misse, A., \& de Diego-Balaguer, R. (2009). Neurophysiological mechanisms involved in language learning in adults. Philos Trans $R$ Soc Lond B Biol Sci, 364(1536), 3711-3735. doi: 10.1098/rstb.2009.0130

Roehrich-Gascon, D., Small, S. L., \& Tremblay, P. (2015). Structural correlates of spoken language abilities: A surface-based region-of interest morphometry study. Brain Lang, 149, 46-54. doi: 10.1016/j.bandl.2015.06.004

Salat, D. H., Buckner, R. L., Snyder, A. Z., Greve, D. N., Desikan, R. S., Busa, E., . . Fischl, B. (2004). Thinning of the cerebral cortex in aging. Cereb Cortex, 14(7), 721-730. doi: 10.1093/cercor/bhh032 
Saur, D., Kreher, B. W., Schnell, S., Kummerer, D., Kellmeyer, P., Vry, M. S., . . . Weiller, C. (2008). Ventral and dorsal pathways for language. Proc Natl Acad Sci U S A, 105(46), 18035-18040. doi: 10.1073/pnas.0805234105

Schlegel, A. A., Rudelson, J. J., \& Tse, P. U. (2012). White matter structure changes as adults learn a second language. J Cogn Neurosci, 24(8), 1664-1670. doi: 10.1162/jocn_a_00240

Scott, S. K., McGettigan, C., \& Eisner, F. (2009). A little more conversation, a little less action--candidate roles for the motor cortex in speech perception. Nat Rev Neurosci, 10(4), 295-302. doi: nrn2603 [pii] 10.1038/nrn2603

Seeley, W. W., Menon, V., Schatzberg, A. F., Keller, J., Glover, G. H., Kenna, H., . . . Greicius, M. D. (2007). Dissociable intrinsic connectivity networks for salience processing and executive control. J Neurosci, 27(9), 2349-2356. doi: 10.1523/JNEUROSCI.5587-06.2007

Simmonds, A. J., Wise, R. J., Dhanjal, N. S., \& Leech, R. (2011). A comparison of sensory-motor activity during speech in first and second languages. $J$ Neurophysiol, 106(1), 470-478. doi: 10.1152/jn.00343.2011

Stein, M., Federspiel, A., Koenig, T., Wirth, M., Strik, W., Wiest, R., . . . Dierks, T. (2012). Structural plasticity in the language system related to increased second language proficiency. Cortex, 48(4), 458-465. doi: 10.1016/j.cortex.2010.10.007

Storsve, A. B., Fjell, A. M., Tamnes, C. K., Westlye, L. T., Overbye, K., Aasland, H. W., \& Walhovd, K. B. (2014). Differential longitudinal changes in cortical thickness, surface area and volume across the adult life span: regions of accelerating and decelerating change. J Neurosci, 34(25), 8488-8498. doi: 10.1523/JNEUROSCI.0391-14.2014

Timarová, Š., Čeňková, I., Meylaerts, R., Hertog, E., Szmalec, A., \& Duyck, W. (2014). Simultaneous interpreting and working memory executive control. Interpreting, 16(2), 139-168. doi: 10.1075/intp.16.2.01tim

Tu, L., Wang, J., Abutalebi, J., Jiang, B., Pan, X., Li, M., . . Huang, R. (2015). Language exposure induced neuroplasticity in the bilingual brain: A followup fMRI study. Cortex, 64, 8-19. doi: 10.1016/j.cortex.2014.09.019

Vandermosten, M., Price, C. J., \& Golestani, N. (2015). Plasticity of white matter connectivity in phonetics experts. Brain Struct Funct. doi: 10.1007/s00429015-1114-8

Wei, G., Zhang, Y., Jiang, T., \& Luo, J. (2011). Increased cortical thickness in sports experts: a comparison of diving players with the controls. PLoS One, 6(2), e17112. doi: 10.1371/journal.pone.0017112

Wenger, E., Schaefer, S., Noack, H., Kuhn, S., Martensson, J., Heinze, H. J., . . . Lovden, M. (2012). Cortical thickness changes following spatial navigation training in adulthood and aging. Neuroimage, 59(4), 3389-3397. doi: 10.1016/j.neuroimage.2011.11.015

Wonderlick, J. S., Ziegler, D. A., Hosseini-Varnamkhasti, P., Locascio, J. J., Bakkour, A., van der Kouwe, A., ... Dickerson, B. C. (2009). Reliability of MRI-derived cortical and subcortical morphometric measures: effects of pulse sequence, voxel geometry, and parallel imaging. Neuroimage, 44(4), 1324-1333. doi: 10.1016/j.neuroimage.2008.10.037

Wong, P. C., Ettlinger, M., Sheppard, J. P., Gunasekera, G. M., \& Dhar, S. (2010). Neuroanatomical characteristics and speech perception in noise in older adults. Ear Hear, 31(4), 471-479. doi: 10.1097/AUD.0b013e3181d709c2 
Yudes, C., Macizo, P., \& Bajo, T. (2011). The influence of expertise in simultaneous interpreting on non-verbal executive processes. Front Psychol, 2, 309. doi: 10.3389/fpsyg.2011.00309

Yudes, C., Macizo, P., \& Bajo, T. (2012). Coordinating comprehension and production in simultaneous interpreters: Evidence from the Articulatory Suppression Effect. Bilingualism-Language and Cognition, 15(2), 329-339. doi: Doi 10.1017/S1366728911000150

Yudes, C., Macizo, P., Morales, L., \& Bajo, M. T. (2013). Comprehension and error monitoring in simultaneous interpreters. doi: 10.1017/S0142716412000112 